Copyright by the Acoustical Society of America. Rogers, C. A., Liang, C., \& Fuller, C. R. (1991). Modeling of shape memory alloy hybrid composites for structural acoustic control. Journal of the Acoustical Society of America, 89(1), 210-220. doi: 10.1121/1.400503

\title{
Modeling of shape memory alloy hybrid composites for structural acoustic control
}

\author{
C. A. Rogers, C. Liang, and C. R. Fuller \\ Smart Materials and Structures Laboratory, Mechanical Engineering Department, Virginia Polytechnic \\ Institute and State University, Blacksburg, Virginia 24061-0238
}

(Received 15 January 1990; accepted for publication 16 May 1990)

\begin{abstract}
Experimental demonstration of active vibration and structural acoustic control using shape memory alloy (SMA) hybrid composites [C. A. Rogers, in Proceedings of the International Congress on Recent Developments in Air and Structure Borne Sound and Vibration (to be published)] has provided the motivation for investigating new control schemes and developing more accurate models. This paper will briefly describe newly developed constitutive models for shape memory alloy actuators and the hybrid material system. A general dynamical model for laminated SMA hybrid composite beams and plates will be presented with several theoretical results. A new structural acoustic model for laminated composite plates [Liang et al., J. Sound Vib. (to be published) ] will be briefly described and the potential for active structural acoustic control using SMA hybrid composites demonstrated by numerical simulation.
\end{abstract}

PACS numbers: $43.40 . \mathrm{Vn}$

\section{INTRODUCTION}

Recent work on novel concepts for both sensing and driving transducers has created strong interest in the active control community (Dimitriadis, et al., 1989). A great deal of work has concentrated around the development and implementation of spatially distributed acutators (Hagood et al., 1989; Rogers et al., 1989) and sensors (Claus et al., 1989). The advantage of distributed control as contrasted to point control has been demonstrated by Meirovitch and Norris (1984). Thus, shape memory alloy hybrid composites have been developed (Rogers and Robertshaw, 1988a) and experimental demonstrations (Rogers, 1990; Saunders et al., 1990) have indicated that this adaptive composite material presents unique paradigms for structural control.

Control of structural acoustic radiation from SMA hybrid composite structures was recently introduced as an important application of SMA hybrid components fibers (Liang et al., 1990; Rogers et al., 1989). Since the characteristic frequencies and mode shape can be modified by activating the embedded SMA fibers, it is possible to modify the sound radiation from the structure (Liang et al., 1989). Figure 1 shows the active modification of mode shapes for a $\left[+45^{\circ} \%-45^{\circ} / 0^{\circ} / 90^{\circ}\right]_{s}$ SMA-graphite epoxy hybrid laminate controlled by active strain energy tuning (ASET) (Rogers et al., 1989). A more detailed discussion is presented in Sec. III. One of the important aspects of how structures radiate sound is the radiation efficiency, which is defined as the ratio of the acoustic power that a structure radiates to power radiated by a piston of equivalent area vibrating with an amplitude equal to the time-spatial average of the structure. Analytical work on simply supported panels with embedded SMA fibers of various configurations has shown that when the actuator fibers are activated then significant modification of the associated acoustic field is achieved (Fuller $e t$ $a l ., 1989)$. This effect has been demonstrated to be due to changes in the panel mode shapes and thus the structural acoustic coupling when the fibers are activated.
Obviously, if the geometric mode shapes of a composite panel can be modified, the natural frequencies of the structure can also be significantly altered. Figure 2 shows a comparison of the first natural frequency of a clamped-clamped graphite-epoxy beam as a function of temperature and a SMA graphite-epoxy hybrid beam (Barker, 1989). The SMA hybrid beam has a nitinol volume fraction of $15 \%$. The first natural frequency of the SMA hybrid beam increases from $21 \mathrm{~Hz}$ at room temperature to $62 \mathrm{~Hz}$ when the actuators are heated to $300^{\circ} \mathrm{F}\left(149{ }^{\circ} \mathrm{C}\right)$. This type of adaptive structural modification also has significant implications on active structural acoustic control. Saunders et al., (1990) experimentally demonstrated frequency placement control for the purpose of minimizing the structurally radiated noise

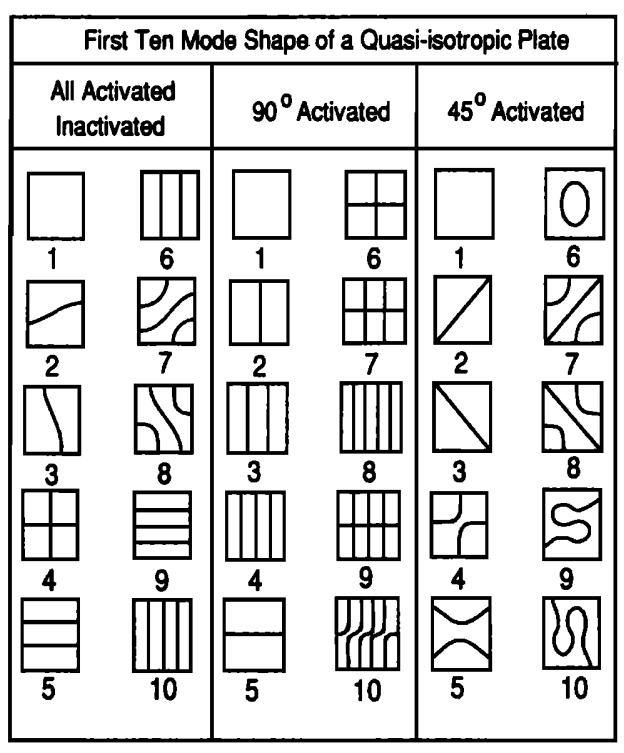

FIG. 1. Comparison of mode shapes of a $\left[+45^{\circ} \%-45^{\circ} / 0 / 90^{\circ}\right]$, square plate using ASET. 


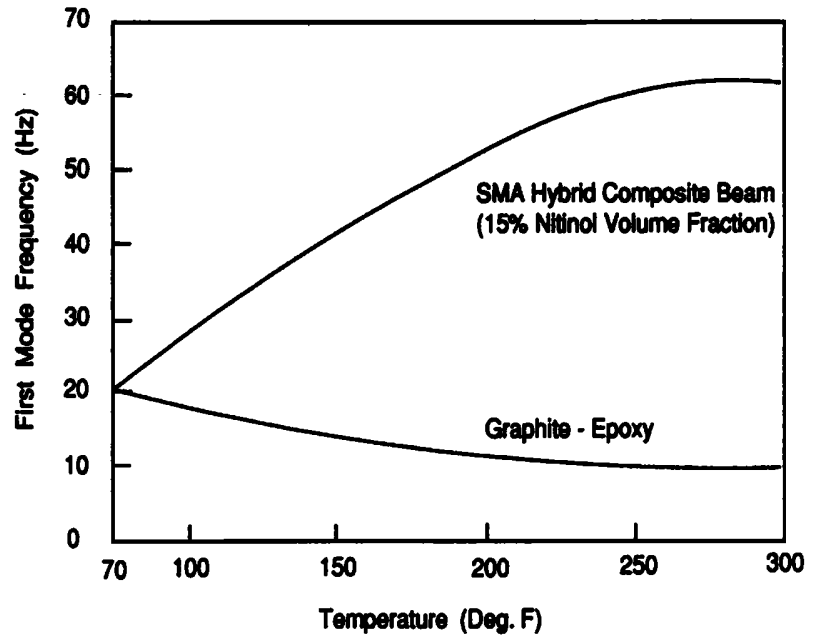

FIG. 2. Comparison of SMA hybrid beam and graphite-epoxy beam first free mode.

from a clamped-clamped beam. Figure 3 shows the results of a minimization experiment in which the beam was excited at $145 \mathrm{~Hz}(n=4$ mode $)$. The reduction in the sound pressure levels were about $25-30 \mathrm{~dB}$. This result, and others, has shown the validity of using SMA hybrid composites to minimize subsonic, structural acoustic radiation.

One of the critical needs to further explore the potential of this unique type of adaptive material system is a refined constitutive and dynamical model. This paper presents the first attempt at developing a complete model beginning with the behavior of the shape memory alloy constituent, including a laminate model and resulting in a unified structural acoustic model for adaptive orthotropic plates.

\section{CONSTITUTIVE RELATIONS}

\section{A. Behavior of shape memory alloy fibers}

A plastically elongated shape memory alloy fiber will tend to return to its original shape when activated by increas-

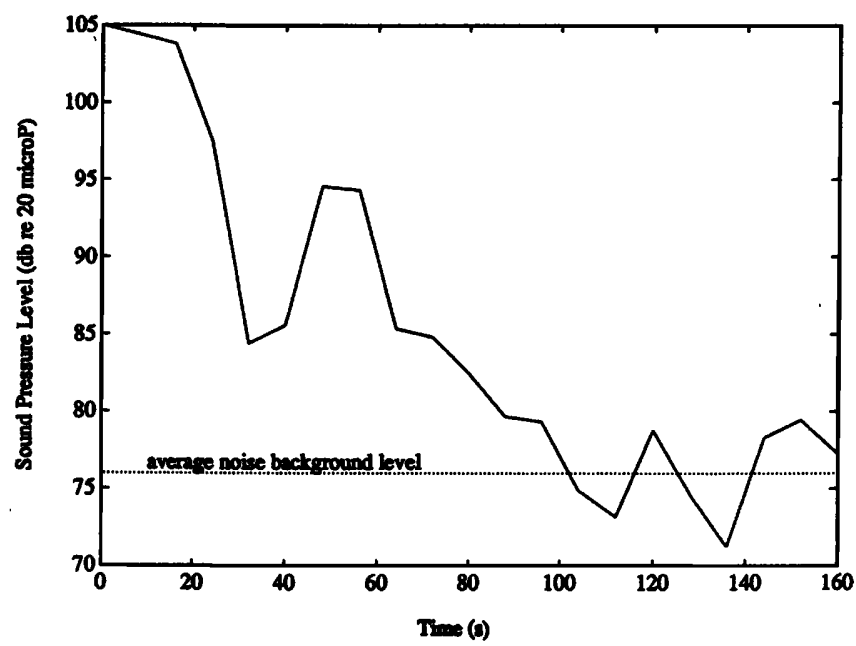

FIG. 3. Structural acoustic control of an SMA hybrid composite beam excited at $145 \mathrm{~Hz}$.

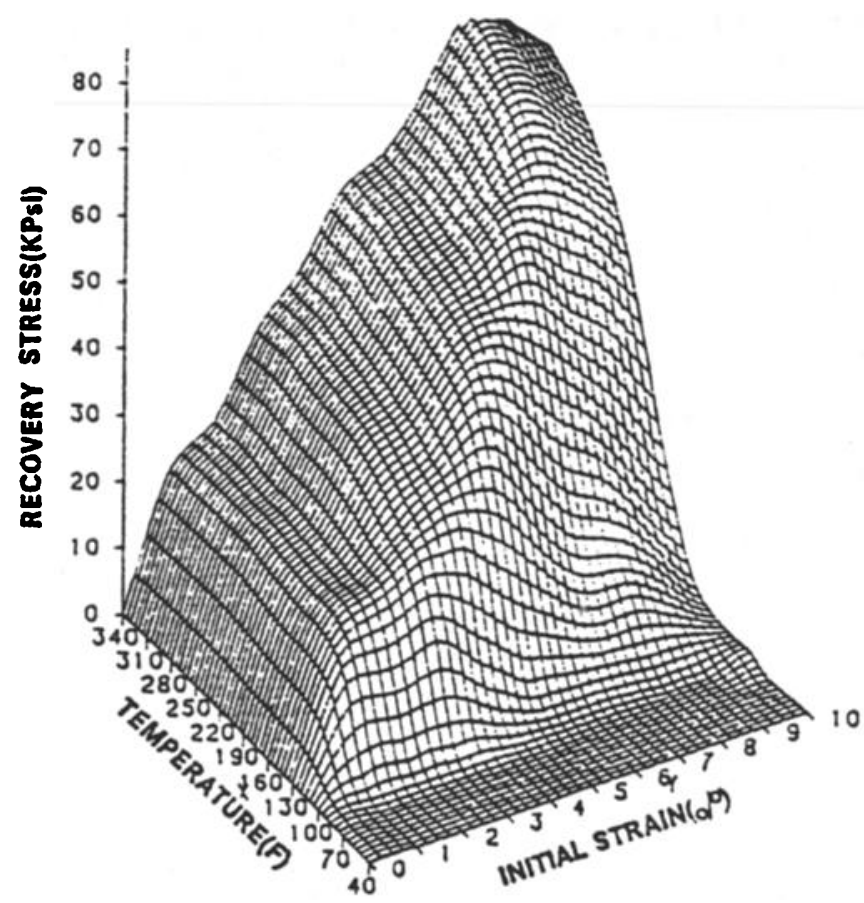

FIG. 4. Stress-strain-activation temperature coupling of nitinol.

ing its temperature above the austenite start temperature; however, the amount of shape change or restoring strain depends on the activation temperature, the initial plastic deformation, $\epsilon_{0}$, and the percent martensite volume fraction. The significant dimensional change is only in the fiber direction because the shape memory phenomena is due to the crystal structure change (twinning). This does not result in a large volume change representative of diffusion phase changes and therefore will not affect the dimension in directions other than the fiber direction beyond Poisson's contractions. Figure 4 (Jia and Rogers, 1989a) shows the typical nonlinear coupling that exists between the recovery stress, initial plastic strain, and the activation temperature.

In shape memory alloy hybrid composites, shape memory alloy fibers, or actuators with an initial plastic strain $\epsilon_{0}$ are embedded in a "matrix." Recall that the "matrix" referred to in this discussion may be a composite material itself, such as graphite-epoxy. The entire material system may then be strained $\epsilon$, which is added to the initial plastic strain $\epsilon_{0}$. The induced global strain $\epsilon$ may be the result of external mechanical loading or from contraction of the material system resulting from activation of the shape memory alloy fibers or actuators. Therefore, the total strain of the actuator $\epsilon_{a}$ will be

$$
\epsilon_{a}=\epsilon_{0}+\epsilon \text {. }
$$

The resulting stress in the SMA fibers upon activation is, therefore, a nonlinear function of initial plastic strain and the activation temperature of the fibers. This relationship can be expressed simply as

$$
\sigma_{a}=f\left(T, \epsilon_{a}\right) \equiv f(\Lambda),
$$

where $\Lambda$ is the state variable of the martensite transformation. 
It will be shown in the next section that the state variables also involve the martensitic fraction $\xi$. When the SMA actuators are employed in our concepts for active dynamic tuning, shape control, structural (i.e., buckling) control, etc., the incremental strain $\epsilon$ will be small. In this paper, we assume this strain is within the elastic limit of the shape memory alloy fibers even though the fibers have been initially plastically elongated. Confining the strain to this linearelastic range simplifies the development and results in the following quasilinear expression relating the actuator stress and strain. Because the restoring stress and Young's modulus both are nonlinear functions of temperature and strain, an * will be used to denote that quantities that are a function of the state variable

$$
E_{a}^{*}=E(\Lambda)
$$

and

$$
\sigma_{r}^{*}=\sigma_{r}\left(T, \epsilon_{0}+\epsilon, \xi\right),
$$

resulting in an expression for the actuator stress as given below

$$
\sigma_{a}=E_{a}^{*} \epsilon+\sigma_{r}^{*} \text {. }
$$

In Eq. (5), $E_{a}^{*}$ is the elastic modulus of the SMA fibers at a given activation temperature $T$. The recovery stress of the SMA fibers at a specified strain and activation temperature is denoted as $\sigma_{r}^{*}$.

Rewriting Eq. (5) to solve for the resulting elastic strain in the actuator as a function of initial strain and activation temperature yields

$$
\epsilon=\sigma_{a} / E_{a}^{*}-\sigma_{r}^{*} / E_{a}^{*} .
$$

To solve for the strain $\epsilon$ in Eq. (6), an iterative method must be used as $\sigma_{r}^{*}$ is a function of strain, $\left(\epsilon_{0}+\epsilon\right)$. The iterative form of Eq. (6) can be written as

$$
\epsilon_{n+1}=\sigma_{a} / E_{a}^{*}-\sigma_{r}^{*}\left(T, \epsilon_{n}\right) / E_{a}^{*} \text {. }
$$

A one-dimensional model incorporating the mechanicalshape memory effect coupling has been formulated and extended to Euler-Bernoulli beams and classical laminated plates theory (Jia and Rogers, 1989b) and to thin shell structures (Jia and Rogers, 1989c).

Figure 5(a) shows the SMA actuator-matrix substructure that has been modeled (Jia and Rogers, 1989b). The stress-strain relationships for this substructure containing a single SMA fiber actuator embedded in a matrix have been developed. In Fig. 5(b), the stresses in a cross section of the substructure is shown. The stress in the matrix $\sigma_{m}$ is generated by two sources, external forces and the actuation of the SMA fibers. The stress $\sigma_{a}$ in the SMA actuators are also induced by two sources, the external mechanical loads and the constriction of the matrix material resulting from the shape recovery after actuation.

One point needs to be made clear concerning the stress components $\sigma_{a}$ and $\sigma_{m}$. Even though two sources are responsible for each of them, the total value of either of them is not the simple superposition of the two effects because the stress-strain relationships are not linear. Therefore, the methods typically used in treating thermoelasticity problems are not suitable in this situation.

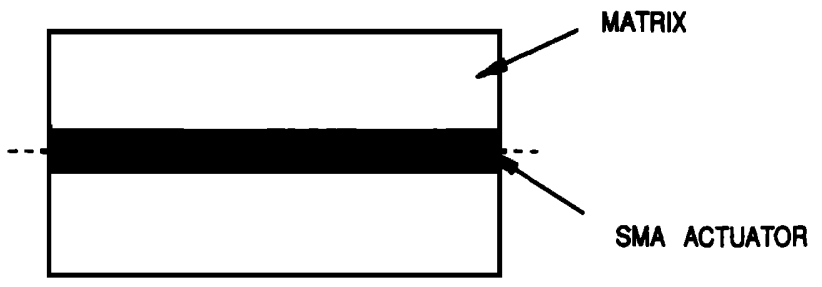

(a)

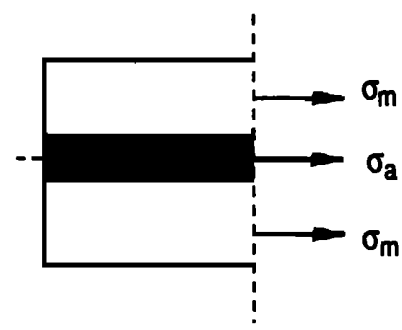

(b)

FIG. 5. Substructure of SMA matrix.

The resultant force in the substructure cross section may be defined as

$$
F=A_{m} \sigma_{m}+A_{a} \sigma_{a},
$$

where $A_{m}$ and $A_{a}$ are the area of matrix and actuator, respectively. The average normal stress is given by

$$
\sigma=F / A=\left(A_{m} \sigma_{m}+A_{a} \sigma_{a}\right) / A \text {. }
$$

The stress-strain relationship derived for the SMA actuators and the linear-elastic "matrix" components are expressed as

$$
\begin{aligned}
& \epsilon=\sigma_{a} / E_{a}^{*}-\sigma_{r}^{*} / E_{a}^{*}, \\
& \epsilon=\sigma_{m} / E_{m} .
\end{aligned}
$$

The stress-strain relationship for the SMA-matrix system is formulated by substituting Eqs. (10) and (11) into Eq. (9) resulting in

$$
\sigma=\left(V_{a} E_{a}^{*}+V_{m} E_{m}\right) \sigma+V_{a} \sigma_{r}^{*},
$$

where $V_{a}$ and $V_{m}$ are the volume fraction of actuators and matrix, respectively.

This one-dimensional model describes the stress-strain behavior of the actuator-matrix material system in the "fiber direction." This model may then be extended to a transversely isotropic (lamina) model by first referring to the one-dimensional model as predicting the behavior of the lamina in the principal (fiber) direction.

One of the fundamental needs in formulating a model to describe the behavior of SMA reinforced hybrid composite lamina is an appropriate micromechanical constitutive relationship. For the purpose of this paper, the micromechanical law used is the rule-of-mixtures. Any micromechanical model could be used and future work will involve investigating the micromechanical response of SMA reinforced composites. However, the rule-of-mixtures relate the global me- 
chanical properties of the lamina to the two constituents, namely the SMA actuators and the "matrix." Recall that the Young's modulus of the actuator fibers is a function of the activation temperature and strain which naturally means that all of the lamina properties will be similarly nonlinear. From the rule-of-mixtures,

$$
\begin{aligned}
& E_{1}^{*}=E_{m}\left(A_{m} / A\right)+E_{a}^{*}\left(A_{a} / A\right), \\
& E_{2}^{*}=A E_{m} E_{a}^{*} /\left(A_{m} E_{a}+A_{a} E_{m}^{*}\right), \\
& v_{12}^{*}=v_{m}\left(A_{m} / A\right)+v_{a}^{*}\left(A_{a} / A\right),
\end{aligned}
$$

and

$$
G_{12}^{*}=A G_{m} G_{a}^{*} /\left(A_{m} G_{a}^{*}+A_{a} G_{m}\right),
$$

where subscript " 1 " refers to the direction of the SMA fibers while "2" denotes the transverse direction of the SMA fibers.

Based on these relations, the temperature-strain-dependent compliance matrix may be written as:

$$
[S]^{*}=\left[\begin{array}{ccc}
S_{11} & S_{21} & 0 \\
S_{12} & S_{22} & 0 \\
0 & 0 & S_{66}
\end{array}\right] *=\left[\begin{array}{ccc}
S_{11}^{*} & S_{21}^{*} & 0 \\
S_{12}^{*} & S_{22}^{*} & 0 \\
0 & 0 & S_{66}^{*}
\end{array}\right],
$$

in which

$$
\begin{aligned}
& S_{11}^{*}=1 / E_{1}^{*}, \\
& S_{12}^{*}=v_{12}^{*} / E_{1}^{*}=-v_{21}^{*} / E_{2}^{*}, \\
& S_{22}^{*}=1 / E_{2}^{*}, \\
& S_{66}^{*}=1 / G_{12}^{*} .
\end{aligned}
$$

The two-dimensional model is a natural extension of the onedimensional model. The general two-dimensional model essentially involves incorporating the tensor transformations appropriate for describing the planar stress-strain behavior of transversely isotropic lamina. However, the most fundamental two-dimensional relationship simply describes the stress-strain behavior in the principal coordinate system. Unlike conventional materials, may they be linear-elastic, elastic-plastic, or possessing any other nonlinear behavior, SMA hybrid composites have a characteristic or tensor quantity that must be incorporated into the basic stressstrain relationship - the shape recovery stress tensor. In the section above, the one-dimensional stress-strain relationship that was derived contained a shape recovery stress term added, as a vector, to the classical rule-of-mixtures "linearelastic" constitutive relationship. Using the resulting temperature-strain lamina properties the following two-dimensional relationships for a transversely isotropic lamina can be expressed in the principal coordinates as (Whitney, 1987):

$$
\left\{\begin{array}{c}
\sigma_{1} \\
\sigma_{2} \\
\sigma_{12}
\end{array}\right\}=\left[\begin{array}{ccc}
Q_{11} & Q_{21} & 0 \\
Q_{12} & Q_{22} & 0 \\
0 & 0 & Q_{66}
\end{array}\right]\left\{\begin{array}{c}
\epsilon_{1} \\
\epsilon_{2} \\
\epsilon_{12}
\end{array}\right\}-\left(\begin{array}{c}
V_{a} \sigma_{r}^{*} \\
0 \\
0
\end{array}\right)
$$

Extending Eq. (18) to describe the mechanical behavior of lamina in the general coordinate system involves performing the tensor transformation and results in

$$
\begin{aligned}
& \left\{\begin{array}{c}
\sigma_{x}^{*} \\
\sigma_{y}^{*} \\
\sigma_{x y}^{*}
\end{array}\right\}=[T]^{-1}\left(\begin{array}{c}
V_{a} \sigma_{r}^{*} \\
0 \\
0
\end{array}\right)=\left\{\begin{array}{c}
c^{2} V_{a} \sigma_{r}^{*} \\
s^{2} V_{a} \sigma_{r}^{*} \\
2 s c B_{a} \sigma_{r}^{*}
\end{array}\right\}, \\
& \left\{\begin{array}{c}
\sigma_{x} \\
\sigma_{y} \\
\sigma_{x y}
\end{array}\right\}=[C]^{*}\left\{\begin{array}{c}
\epsilon_{x} \\
\epsilon_{y} \\
\epsilon_{x y}
\end{array}\right\}+\left\{\begin{array}{c}
\sigma_{x}^{*} \\
\sigma_{y}^{*} \\
\sigma_{x y}^{*}
\end{array}\right\},
\end{aligned}
$$

where " $c$ " and " $s$ " are the cosine and sine of the transformation angle, respectively. Equation (20) is the stress-strain relationship for a single layer, or lamina, of SMA reinforced composite. Each lamina of a laminate will therefore have a different effective stiffness and resulting stress-strain relationship depending on: the fiber/actuator orientation, the actuator volume fraction, and the activation temperature of each lamina. For laminate analysis, each lamina must be first evaluated as described above and then denoted by using the superscript $(k)$ to identify individual plys.

\section{B. Constitutive model for shape memory alloys}

The reversible thermoelastic martensitic phase transformation of shape memory alloys is the fundamental physical transduction mechanism utilized for active control. However, the constitutive models that have been proposed and published in the last 30 years have lacked several important fundamental elements and capabilities which are essential for the active control schemes described in this paper. As a result, a complete unified one-dimensional constitutive model was developed that allows for rigorous investigations of many of the important material characteristics involved with the internal phase transformation of shape memory alloys (Liang and Rogers, 1990). These characteristics include energy dissipation in the material that governs the damping behavior, stress-strain-temperature relations for pseudoelasticity, and the shape memory effect.

There are basically two approaches generally considered for developing a constitutive relation for any material. One is the macroscopic phenomenological method that requires a significant amount of experimental work; the other is the microscopic physical method that derives the constitutive relation from fundamental physical concepts. The phenomenological approach is often used in engineering practice but can rarely explain the physics behind its material behavior or character. The microscopic physical method can successfully provide the fundamental explanations to different experimental phenomena, however, numerical predictions and simulations are often complex and distant from phenomenological observations.

Müller $(1979,1985)$ has developed a model based on shape memory phenomenology, thermodynamics, and statistical physics. Tanaka and Nagaki (1982) developed a model similar to Müller's in which the phase transformation is governed by the minimization of the Helmholtz free energy. Tanaka's model uses the energy equation and the Clausius-Duhem inequality to describe the hysteresis associated with the phase transformation. This approach has been extended (Tanaka, 1986) and used to describe and simulate superelasticity (Tanaka and Iwasaki, 1985), describe the 
one-dimensional phase transformation mechanics (Tanaka, 1986), pseudoelasticity associated with stress-induced martensitic transformation (Sato et al., 1986) and the damping behavior of shape memory alloys (Sato and Tanaka, 1988).

Liang and Rogers (1990) have developed a similar model to Tanaka's with the intent of evaluating the variation of the Young's modulus, restoring stress, damping capacity, and other factors associated with the martensitic transformation and essential for active control simulation and model implementation. In this model the state variables for the material are stress, strain, temperature, and extent of the transformation $\xi$, which is defined as the martensitic fraction and schematically shown in Fig. 6 . Since only three of these variables are independent, the state variable is defined as

$$
\Lambda \equiv(\bar{\epsilon}, T, \xi) \text {. }
$$

The Helmholtz free energy is defined as

$$
\Psi=E-T S,
$$

in which the energy $E$ and entropy $S$ is a function of the state variable $\Lambda$. Because the shape memory effect involves large deformations, the Green strain $\bar{\epsilon}$ and the Piola-Kirchoff stress $\bar{\sigma}$ are used resulting in a new form of the ClausiusDuhem inequality

$$
\begin{aligned}
\left(\frac{\bar{\sigma}}{\rho_{0}}\right. & \left.-\frac{\partial \Psi}{\partial \bar{\epsilon}}\right) \dot{\bar{\epsilon}}-\left(S+\frac{\partial \Psi}{\partial T}\right) \dot{T} \\
& -\frac{\partial \Psi}{\partial \xi} \dot{\xi}-\frac{1}{\rho_{0} T} \frac{\rho_{0}}{\rho} q f^{-1} \frac{\partial T}{\partial X} \geqslant 0,
\end{aligned}
$$

where $f$ is the deformation gradient and $\rho_{0}$ is the density in the reference configurations. From the thermodynamics of continuous media, the coefficients of $\dot{\bar{\epsilon}}$ and $\dot{T}$ should vanish. Therefore, the inequality of Eq. (23) provides the mechanical constitutive equation as

$$
\bar{\sigma}=\rho_{0} \frac{\partial \Psi}{\partial \bar{\epsilon}}=\sigma(\bar{\epsilon}, T, \xi) .
$$

From the above equation, the rate form of the mechanical constitutive equation is obtained as

$$
\dot{\bar{\sigma}}=\frac{\partial \sigma}{\partial \bar{\epsilon}} \dot{\bar{\epsilon}}+\frac{\partial \sigma}{\partial T} \dot{T}+\frac{\partial \sigma}{\partial \xi} \dot{\xi}=D \dot{\bar{\epsilon}}+\Theta \dot{T}+\Omega \dot{\xi}
$$

where $D$ is the Young's modulus, $\Theta$ the thermoelastic tensor,

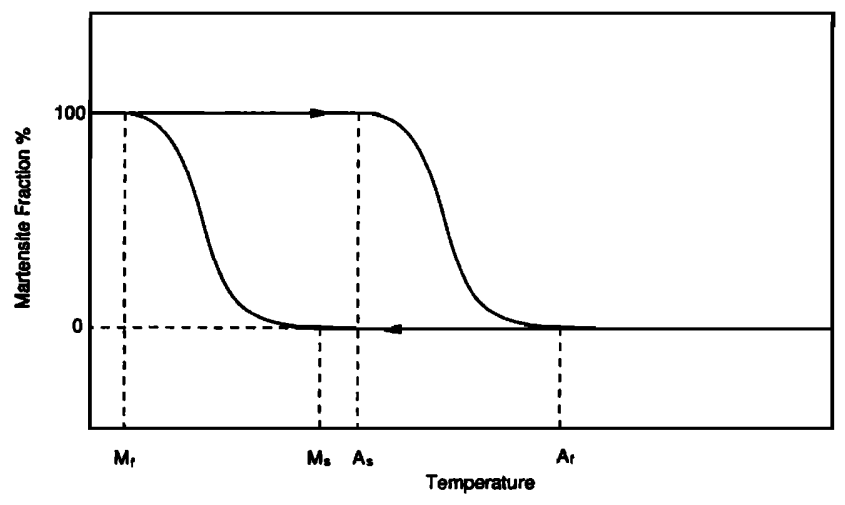

FIG. 6. Martensite fraction versus temperature. and $\Omega$ the transformation tensor, a metallurgical quantity. These material properties derived from thermomechanics are given as

$D=\rho_{0} \frac{\partial^{2} \Psi}{\partial \bar{\epsilon}^{2}}, \quad \Theta=\rho_{0} \frac{\partial^{2} \Psi}{\partial \bar{\epsilon} \partial T}, \quad \Omega=\rho_{0} \frac{\partial^{2} \Psi}{\partial \bar{\epsilon} \partial \xi}$.

Whereas Tanaka (1986) described the relation of martensite fraction and temperature during the phase transformation in an exponential form, Liang and Rogers (1990) used a cosine function. Tanaka's work has been primarily studying the stress-strain behavior of SMA materials qualitatively and the current need in active structural control is a quantitative model with a minimal amount of experimental material constants needed and with constants that are easy to obtain.

Shape memory alloys may be used in several geometric forms and utilize various transduction principles for active control applications. One approach is to use SMA wire or fiber as distributed force actuators. SMA force actuators are first elongated at a low temperature and then unloaded to generate some martensitic residual strain; upon heating the wire, the martensitic residual strain will be restored. The shape memory recovery generally falls into one of the following categories. First is free recovery in which there is no external load on the wire, and therefore, no work. The second category includes fully restrained "recovery." In this case, the martensitic residual strain of the SMA wire is restrained from being restored to its original length. This restraint causes large internal "recovery" stresses to be generated. The third category is called controlled recovery. In this case, some residual martensitic strain is restored (the restored part is called the recovery strain ), but the wire is still under some tension which is required to prevent full recovery of the residual strain.

For active vibration and structural acoustic control using SMA hybrid composites, the most important class of problems is the controlled recovery case. Controlled recovery is defined as the shape memory recovery process during which some recovery strain is allowed during the heating and cooling cycle, such as the recovery strain that is observed when an embedded SMA fiber is activated in a composite panel. The recovery strain, and therefore the recovery stress, is dependent on the spring rate or modulus of the constraining material.

In order to develop a time-independent model rate form of the constitutive equation, the governing equation, Eq. (25), is integrated with respect to time and yields what is called the unified constitutive relation:

$$
\sigma-\sigma_{0}=D\left(\epsilon-\bar{\epsilon}_{0}\right)+\Theta\left(T-T_{0}\right)+\Omega\left(\xi-\xi_{0}\right),
$$

where the subscript "0" denotes initial conditions. This unified equation is rewritten for controlled recovery as

$$
\bar{\sigma}^{r}-\bar{\sigma}_{0}^{r}=D\left(\bar{\epsilon}^{r}-\bar{\epsilon}_{0}^{r}\right)+\Theta\left(T-T_{0}\right)+\Omega\left(\xi-\xi_{0}\right) .
$$

Assuming the loading is proportional to the recovery strain (which applies to most SMA actuators) and modeling the actuator system as a SMA wire of cross section $S$, in parallel with a spring of spring rate $k$, length $L$, the stress-strain relation for the spring is 


$$
\sigma^{r}-\sigma_{0}^{r}=(k L / S)\left(\bar{\epsilon}_{\mathrm{res}}-\bar{\epsilon}^{r}\right),
$$

where $\bar{\epsilon}_{\text {res }}$ is the residual strain. Substituting this expression into the constitutive equation, Eq. (28), yields

$$
(1+s D / k L)\left(\bar{\sigma}^{r}-\bar{\sigma}_{0}^{r}\right)=\Theta\left(T-T_{0}\right)+\Omega\left(\xi-\xi_{0}\right) .
$$

The equation above can now be used to determine the recovery stress and/or recovery strain for any specific temperature and path by first calculating an initial value of the martensite fraction using the phenomenological cosine expressions developed by Liang and Rogers (1990), which are functions of the stress and therefore results in an iterative solution.

This improved model more accurately predicts the experimental observations using the most basic material constants required to completely describe the behavior of shape memory alloys. This model greatly improves the accuracy and reliability of simulations of structural control using SMA factor actuators.

\section{PLATE DYNAMICS RELATIONS}

\section{A. Formulation}

A symmetric SMA reinforced laminate may be described by the following governing equation (Rogers and Robertshaw, 1988b):

$$
\begin{gathered}
D_{11}^{*} \frac{\partial^{4} W}{\partial x^{4}}+4 D_{16}^{*} \frac{\partial^{4} W}{\partial x^{3} \partial y}+2\left(D_{12}^{*}+2 D_{66}^{*}\right) \frac{\partial^{4} W}{\partial x^{2} \partial y^{2}} \\
+4 D_{26}^{*} \frac{\partial^{4} W}{\partial x \partial y^{3}}+D_{22}^{*} \frac{\partial^{4} W}{\partial y^{4}}+\bar{N}_{x}^{*} \frac{\partial^{2} W}{\partial x^{2}}+\bar{N}_{y}^{*} \frac{\partial^{2} W}{\partial y^{2}} \\
+2 \bar{N}_{x y}^{*} \frac{\partial^{2} W}{\partial x \partial y}+C_{D} \frac{\partial W}{\partial t}+\rho \frac{\partial^{2} w}{\partial t^{2}}=q(x, y) e^{i \omega t},
\end{gathered}
$$

where $C_{D}$ is a damping coefficient, $\rho$ is mass per unit area, and $q(x, y) e^{i \omega t}$ is the external forcing function. Here, $\bar{N}^{*}$ 's are the recovery stress resultants. After activation of the SMA actuators, these recovery force resultants will exist, and at the same time, $D^{*}$ 's will change as a result of activation. If the SMA actuators are embedded in a lamina without initial plastic strain, activation of the SMA can only change the lamina's properties, i.e., $D^{*}$ s.

\section{Assume}

$$
W=w(x, y) e^{i \omega t}
$$

for the steady-state solution of the plate. By substituting the above equation into Eq. (7), we obtain

$$
\begin{aligned}
D_{11}^{*} & \frac{\partial^{4} w}{\partial x^{4}}+4 D_{16}^{*} \frac{\partial^{4} w}{\partial x^{3} \partial y}+2\left(D_{12}^{*}+2 D_{66}^{*}\right) \frac{\partial^{4} w}{\partial x^{2} \partial y^{2}} \\
& +4 D_{26}^{*} \frac{\partial^{4} w}{\partial x \partial y^{3}}+D_{22}^{*} \frac{\partial^{4} w}{\partial y^{4}}+\bar{N}_{x}^{*} \frac{\partial^{2} w}{\partial x^{2}}+\bar{N}_{y}^{*} \frac{\partial^{2} w}{\partial y^{2}} \\
& +2 \bar{N}_{x y}^{*} \frac{\partial^{2} w}{\partial x \partial y}+C_{D} i \omega w-\rho \omega^{2} w=q(x, y) .
\end{aligned}
$$

The typical boundary conditions for a simply supported rectangular plate are used and the Rayleigh-Ritz method is used in which the assumed solution for the energy expression using the separation of variables, is

$$
w=\sum_{m=1}^{M} \sum_{n=1}^{N} A_{m n} X_{m}(x) Y_{n}(y) .
$$

Substituting this series into the energy expression, the set of simultaneous equations result. The linear simultaneous equations are then rewritten in matrix form as

$$
[K]\{A\}=\{q\} .
$$

For a simply supported plate, the double sine series in conjunction with the assumed solution given by Eq. (34) satisfies both the geometry and natural boundary conditions:

$$
\begin{aligned}
& X_{m}(x)=\sin (m \pi x / a), \\
& Y_{n}(y)=\sin (n \pi y / b),
\end{aligned}
$$

where " $a$ " and " $b$ " are the dimensions of the plate in $x$ and $y$ direction, respectively.

At this point, the unknown coefficients $\{A\}$ are determined and the deflections calculated using the assumed solution of Eq. (32). Because of the approximate nature of the Ritz method, care must be taken to insure convergence of the solution and that the coupling terms, $D_{16}$ and $D_{26}$, are accounted for appropriately (Whitney, 1987).

The values of $M$ and $N$ must be chosen to satisfy the convergence requirement. In our calculation, we found that $M=7$ and $N=7$ insures convergence.

\section{B. Bending of simply supported plates}

The pure pending of a plate is a static problem. In Eq. (33) the inertial term and damping term should vanish. Using the procedure as described above, we can get the same form of the system equations:

$$
[K]\{A\}=\{q\},
$$

where

$$
q_{(m+1) N+n}=\int_{0}^{a} \int_{0}^{b} q X_{m} Y_{n} d x d y .
$$

The solution from the linear system can be substituted back into Eq. (37) to find the deflection.

\section{Free vibration of simply supported plates}

In the free vibration case, the external forcing term does not exist. To find the system's undamped natural frequency, the damping coefficients $C_{D}$ are to be zero.

Once again, we have the linear equation system:

$[K]\{A\}=\{0\}$.

For a simply supported plate, the above equation can be expressed as

$$
\left([\bar{K}]-(a b \rho / 4) \omega^{2}[I]\right)\left\{A_{t}\right\}=\{0\} .
$$

The eigenvalues $\lambda_{i}$ of $[\bar{K}]$ are then used to get the natural frequencies of the system as following:

$$
\omega_{i}=\sqrt{4 \lambda_{i} / a b \rho} .
$$

The coefficients $\{A\}$ gives the mode shape corresponding to the frequency $\omega_{i}$ which can be found from Eq. (40).

\section{THEORETICAL RESULTS}

A "standard" plate is used and referred to in the following results and discussion in order to demonstrate the au- 
thority of control while keeping as many structural parameters constant as possible. The "matrix" material used is graphite/epoxy. The rectangular plate $(20 \times 15 \times 0.1 \mathrm{in}$. $)$ is symmetric and quasi-isotropic using a $\left[+45^{\circ} /-45^{\circ} / 90^{\circ} / 0^{\circ}\right]_{s}$ lay-up. The results are nondimensionalized wherever possible which generalizes the discussion and reduces the significance of the selected plate size. The plate is simply supported and restrained to eliminate any in-plane displacements.

SMA reinforced composites can be manufactured and designed several ways with the actual lay-up sequence being an integral tailoring parameter for effective structural control (Barker, 1989). In all of the formulations, analysis, and experimental results presented in this paper, the structures are symmetric. There are two primary lay-up schemes used to embed the SMA fiber actuators. In the first, the nitinol fibers are embedded to coincide with the principle material direction of the "matrix" composite lamina and is referred to as embedding in the $E_{11}$ direction. Likewise, the second technique is to embed the actuator fibers in the $E_{22}$ direction of the lamina so as to create a more significant change in a 'stiffness' term of the lamina. Recall that the Young's modulus of nitinol is typically controllable between 4 and $16 \mathrm{Mpsi}$, which is significantly lower than $E_{11}$ for graphite/epoxy.

The variations and modifications of the mode shapes for a $\left[+45^{\circ} /-45^{\circ} / 0^{\circ} / 90^{\circ}\right]_{s}$ square plate controlled by ASET are shown in Fig. 1. For the case in which 90-deg laminae are activated, the mode shapes change considerably from the inactivated plate. The fourth mode shape of the 90-deg ply activated plate is essentially the 10th mode shape of the inactivated plate. The ability to change the mode shape of a structure has significant impact on the control of structural acoustic radiation. For example, the third mode of the 90 deg activated plate has a higher radiation efficiency than the third mode of the inactivated plate, which will result in a considerably different transmission loss profile as will be discussed later in this paper. Currently, a design and control methodology is being developed to demonstrate the ability to significantly alter the mode shape of a structure without necessarily changing the resonant or natural frequency.

\section{A. Structural acoustic theory}

Before discussing the analysis of transmission of sound through composite plates, it is first important to understand the modal behavior of orthotropic plates. Liang et al. (1990) presented a detailed treatment of free vibration frequency and mode shape expression of orthotropic plates in order to perform the structural acoustic analysis. In this work, a rectangular simply supported orthotropic plate was considered using the Raleigh-Ritz solution method. Using this method, the $k$ th normalized mode shape $\phi_{k}$ is given by

$$
\phi_{k}=\sum_{m=1}^{M} \sum_{n=1}^{N} A_{m n}^{k} \sin \frac{m \pi x}{a} \sin \frac{n \pi y}{b} .
$$

Unlike isotropic simply supported plates, the mode shape of a composite laminate consists of more than one term, here we call the double $\sin t$ term, $\sin (m \pi x / a) \sin (n \pi y / b)$, the characteristic mode and summation of all the characteristic modes weighted by $A_{m n}^{k}, \phi_{k}$, the composite mode. The orth- ogonality of the mode shapes can be described by

$$
\int_{0}^{a} \int_{0}^{b} \phi_{i} x \phi_{j} d x d y= \begin{cases}0, & i \neq j \\ a b / 4, & i=j .\end{cases}
$$

Activating the SMA fibers embedded in the laminate can change both the natural frequencies and the mode shapes. To determine how much the mode shape has been changed, let $\bar{\Phi}$ be the mode shape of an inactivated plate and $\Phi$ be that of the activated plate (here all the variables with a bar correspond to the inactivated plate). The $k$ th inactivated mode $\bar{\Phi}_{k}$ can be expressed as

$$
\bar{\Phi}_{k}=\sum_{m=1}^{M} \sum_{n=1}^{N} \bar{A}_{m n}^{k} \sin \frac{m \pi x}{a} \sin \frac{n \pi y}{b} .
$$

Mathematically, $\Phi_{k}$ in Eq. (42) can also be expressed as

$$
\Phi_{k}=\sum_{i=1}^{M x N} C_{i}^{k} \bar{\Phi}_{i} \text {. }
$$

Note, the sum should be infinite, approximation using first $M \times N$ nodes is used here.

The activation mode transformation coefficient $C_{i}^{k}$ can be calculated by using Eq. (43), resulting in

$$
C_{i}^{k}=\frac{\sum_{m=1}^{M} \sum_{n=1}^{N} \bar{A}_{m n}^{i} A_{m n}^{k}}{\sum_{m=1}^{M} \Sigma_{n=1}^{N} \bar{A}_{m n}^{i} \bar{A}_{m n}^{i}} .
$$

Because the composite mode has been normalized, the denominator of the above equation equals to one. By evaluating $C_{i}^{k}$, it is possible to determine how the mode shape changes and how much it changes compared with the mode shapes of the inactivated plate. For a simply supported plate excited by incident sound pressure, the displacement $w(x, y)$ can also be written in terms of mode shapes of the inactivated plate, $\bar{\Phi}_{k}$, using the following form regardless of whether the plate is activated or not:

$$
w(x, y)=\sum_{i=1}^{\infty} B_{i} \bar{\Phi}_{i} .
$$

The first $M \times N$ mode shapes are used to approximate the infinite series. The displacement mode coefficient $B_{i}$ in the equation above can be expressed as

$$
B_{i}=\frac{\sum_{m=1}^{M} \Sigma_{n=1}^{N} A_{m n} \bar{A}_{m n}^{i}}{\sum_{m=1}^{M} \Sigma_{n=1}^{N} \bar{A}_{m n}^{i} \bar{A}_{m n}^{i}} .
$$

Similarly, the denominator in the above equation is equal to one. Here, $B_{i}$ indicates the influence of the $i$ th mode on the displacement amplitude.

The analysis of transmission of sound through SMA hybrid composites begins with the fundamental analysis scheme presented by Roussos (1985). In this case, an acoustic plane wave is incident on the plate at an angle $\theta_{i}$. The incident acoustic wave excites the plate and causes transmission of acoustic energy to the other side of the plate. If a light acoustic fluid such as air is assumed, it then becomes reasonable to assume that the pressure forcing function on the incident side of the plate is the blocked pressure $q(x, y)$ which is given by

$$
\begin{aligned}
q(x, y)= & 2 P_{i} \exp \left[-(\omega i / c)\left(x \sin \theta_{i} \cos \phi_{i}\right.\right. \\
& \left.\left.+y \sin \theta_{i} \sin \phi_{i}\right)\right],
\end{aligned}
$$


where $\omega$ is steady-state excitation radian frequency, $c$ is the speed of sound, $\theta_{i}$ is the polar incidence angle, $\phi_{i}$ is the asimuthal incidence angle, and $P_{i}$ is the real incident pressure magnitude. The modal components of the transverse loads,

$q_{s}$, may then be written as

$$
q_{s}=2 P_{i} \bar{I}_{m} \bar{I}_{n},
$$

where

$$
\begin{aligned}
& \bar{I}_{m}=\left\{\begin{array}{cc}
-(i / 2) \operatorname{sgn}\left(\sin \theta_{i} \cos \theta_{i}\right) & \left(m \pi^{2}\right)=\left[\sin \theta_{i} \cos \phi_{i}(\omega a / c)\right]^{2}, \\
\frac{m \pi\left\{1-(-1)^{m} \exp \left[-i \sin \theta_{i} \cos \phi_{i}(\omega a / c)\right]\right\}}{(m \pi)^{2}-\left[\sin \theta_{i} \cos \phi_{i}(\omega a / c)\right]^{2}} & (m \pi)^{2} \neq\left[\sin \theta_{i} \cos \phi_{i}(\omega a / c)\right]^{2},
\end{array}\right. \\
& \bar{I}_{n}=\left\{\begin{array}{cc}
-(i / 2) \operatorname{sgn}\left(\sin \theta_{i} \sin \theta_{i}\right) & (n \pi)^{2}=\left[\sin \theta_{i} \sin \phi_{i}(\omega b / c)\right]^{2}, \\
\frac{n \pi\left\{1-(-1)^{n} \exp \left[-i \sin \theta_{i} \sin \phi_{i}(\omega b / c)\right]\right\}}{(n \pi)^{2}-\left[\sin \theta_{i} \sin \phi_{i}(\omega b / c)\right]^{2}} & (n \pi)^{2} \neq\left[\sin \theta_{i} \sin \phi_{i}(\omega b / c)\right]^{2} .
\end{array}\right.
\end{aligned}
$$

Solving the resulting set of simultaneous equations which result from the Rayleigh-Ritz approximation to governing equation of motion yields the displacement coefficients, $A_{m n}$, from which the transmitted pressure can be expressed using the Rayleigh integral approach as

$$
P_{t}(r, \theta, \phi)=\frac{-\omega^{2} \rho a b}{2 \pi r} \exp \left\{i \omega\left[t-\frac{r}{c}-\frac{\sin \theta}{2 c}(a \cos \phi+b \sin \phi)\right]\right\} \sum_{m=1}^{M} \sum_{n=1} N A_{m n} I_{m} I_{n},
$$

where

$$
\begin{gathered}
I_{m}=\left\{\begin{array}{cc}
-(i / 2) \operatorname{sgn}(\sin \theta \cos \theta) & (m \pi)^{2}=[\sin \theta \cos \phi(\omega a / c)]^{2}, \\
\frac{m \pi\left\{1-(-1)^{m} \exp [i \sin \theta \cos \phi(\omega a / c)]\right\}}{(m \pi)^{2}-[\sin \theta \cos \phi(\omega a / c)]^{2}} & (m \pi)^{2} \neq[\sin \theta \cos \phi(\omega a / c)]^{2},
\end{array}\right. \\
I_{n}=\left\{\begin{array}{cc}
-(i / 2) \operatorname{sgn}(\sin \theta \sin \theta) & (n \pi)^{2}=[\sin \theta \sin \phi(\omega b / c)]^{2}, \\
\frac{n \pi\left\{1-(-1)^{n} \exp [i \sin \theta \sin \phi(\omega b / c)]\right\}}{(n \pi)^{2}-[\sin \theta \sin \phi(\omega b / c)]^{2}} & (n \pi)^{2} \neq[\sin \theta \sin \phi(\omega b / c)]^{2},
\end{array}\right.
\end{gathered}
$$

$r$ is the radial distance from a point in the far field to the center of the plate, $\theta$ is the polar angle location of a point in the far field, and $\phi$ is the azimuthal angle location of a point in the far field. The above equations are explained in greater detail in Liang et al. (1990) and Roussos (1985).

\section{B. Numerical case studies}

In the numerical case studies presented below, a composite plate of $1.1 \mathrm{~m}$ by $0.8 \mathrm{~m}$ by $0.008 \mathrm{~m}$ is used with the objective being to determine the directivity pattern, transmission loss, and radiation efficiency, etc. (Liang, Rogers, and Fuller, 1990). The stacking sequence of the plate is $\left[0^{\circ} /-45^{\circ} / 45^{\circ} / 90^{\circ}\right]_{s}$, resulting in a quasi-isotropic plate. The plate is assumed to be made of equal-thickness graphite/epoxy and nitinol/epoxy laminae. The nitinol/epoxy lamina is considered to be the top and bottom plys of the laminate. The nitinol/epoxy lamina is assumed to have a $40 \%$ Nitinol fiber volume fraction which yields an actuator fiber volume fraction of $10 \%$ for the entire plate. It is assumed that the recovery stress of the embedded SMA fibers on acti- vation is $280 \mathrm{MPa}$. The recovery stress is related to the initial strain, the recovery stress-strain relation of the SMA fiber, the curing process of the composite plate which could cause stress relaxation, and the boundary conditions of the plate. These issues are presently being investigated in detail.

The incident acoustic plane wave has a polar angle of 45 $\mathrm{deg}$ and azimuthal angle of $0 \mathrm{deg}$. The damping coefficient $\xi$ is chosen to be 0.01 , which corresponds to most light fluids. The damping constant and the coefficient has the following relation:

$$
C_{D}=2 m_{p} \omega \xi
$$

Table I contains the calculated natural frequencies for the first ten natural frequencies of the inactivated and fully activated plate. The first natural frequency has been increased

\begin{tabular}{|c|c|c|c|c|c|c|c|c|c|c|}
\hline & \multicolumn{10}{|c|}{ Natural frequencies $(\mathrm{Hz})$} \\
\hline & 1 & 2 & 3 & 4 & 5 & 6 & 7 & 8 & 9 & 10 \\
\hline Inact. & 41.3 & 82.8 & 114.8 & 144.4 & 166.9 & 224.0 & 233.7 & 245.5 & 290.7 & 317.9 \\
\hline Act. & 71.5 & 129.7 & 146.6 & 203.4 & 239.5 & 246.9 & 296.5 & 322.4 & 355.4 & 403.4 \\
\hline
\end{tabular}
by approximately $73 \%$ upon activation of the embedded SMA fibers. The change of mode shape can be described numerically as defined by Eqs. (45) and (46). In Table II, the activation mode transformation coefficient $C_{i}^{*}$ for the first ten modes are given showing the change in the activated

TABLE I. Change of the first ten natural frequencies $(\mathrm{Hz})$. 
TABLE II. Change of the first ten mode shapes.

\begin{tabular}{|c|c|c|c|c|c|c|c|c|c|c|}
\hline \multirow[t]{2}{*}{ Act. } & \multicolumn{10}{|c|}{ Inactivated modes } \\
\hline & 1 & 2 & 3 & 4 & 5 & 6 & 7 & 8 & 9 & 10 \\
\hline 1 & 1.00 & 0.000 & 0.000 & 0.005 & 0.009 & -0.001 & 0.000 & 0.000 & 0.000 & 0.002 \\
\hline 2 & 0.000 & 0.327 & 0.945 & 0.000 & 0.000 & 0.000 & 0.000 & 0.010 & 0.006 & 0.000 \\
\hline 3 & 0.000 & 0.945 & -0.327 & 0.000 & 0.000 & 0.000 & 0.000 & 0.007 & -0.004 & 0.000 \\
\hline 4 & -0.011 & 0.000 & 0.000 & 0.505 & 0.861 & 0.028 & 0.051 & 0.000 & -0.000 & 0.000 \\
\hline 5 & 0.000 & 0.000 & 0.000 & 0.856 & -0.493 & -0.143 & -0.151 & 0.000 & -0.000 & 0.019 \\
\hline 6 & 0.001 & 0.000 & 0.000 & 0.105 & -0.120 & 0.989 & 0.987 & 0.000 & 0.000 & -0.014 \\
\hline 7 & 0.000 & -0.014 & 0.000 & 0.000 & 0.000 & 0.000 & 0.000 & 0.600 & 0.281 & 0.000 \\
\hline 8 & 0.000 & 0.006 & -0.009 & 0.000 & 0.000 & 0.000 & 0.000 & -0.038 & 0.945 & 0.000 \\
\hline 9 & 0.000 & 0.002 & 0.009 & 0.000 & 0.000 & 0.000 & 0.000 & -0.799 & 0.167 & 0.000 \\
\hline 10 & 0.000 & -0.001 & 0.000 & 0.000 & 0.000 & 0.000 & 0.000 & 0.008 & -0.028 & 0.000 \\
\hline
\end{tabular}

mode shapes compared to the inactivated mode shapes. For example, the eighth activated mode shape is almost identical to the ninth inactivated mode shape.

It is also important to note that the $k$ th activated mode shape is not necessarily dominated by the $k$ th inactivated mode shape. In fact, for the problem given, only the sixth inactivated mode remains dominant in corresponding activated mode. However, typically the $k$ th activated mode is dominated by the $k$ th inactivated mode and its "near-by" modes.

\section{Directivity pattern}

It can be seen that the directivity pattern is governed mostly by the composite mode of the plate which could be changed by tuning the recovery stress of the embedded SMA fibers. The results presented here are for an incident sound wave at $\theta_{i}=45^{\circ}$ and $\phi_{i}=0^{\circ}$ for two frequencies of 100 and $220 \mathrm{~Hz}$. Figure 7 shows the variation of the transmitted intensity with polar angle $\theta$ across the $\phi=0^{\circ}, \phi=180^{\circ}$ diagonal for both an activated and inactivated plate at $100 \mathrm{~Hz}$.

From Eqs. (47) and (48), the modes which play the dominant role in the response $w(x, y)$ can be easily obtained. For the inactivated plate, $B_{1}$ and $B_{2}$ are found to be the same and both have the largest magnitude. Because the second mode has a 180-deg phase difference from the first mode in the $x>a / 2$ region, the superimposed $w(x, y)$ is relatively small. Therefore, the intensity pattern appears as in the figure with a moved center to the left. However, for the activated plate, the $B_{1}$ is the largest term and is ten times larger than the others. Therefore, the plate is vibrating more like its first mode, or a monopole, and the center of the pattern is located about the center of the plate with relatively larger magnitudes. Figure 8 shows the variation of the transmitted intensity with polar angle for $\phi=0^{\circ}$ and $\phi=180^{\circ}$ for both an activated and inactivated plate at $220 \mathrm{~Hz}$. Once again, the intensity pattern is changed significantly because of the change of $w(x, y)$. It is also clear that as the frequency increases, more transmitted sound becomes concentrated at the transmitted angle which is closed to the incident angle. It can also be noted that the directivity pattern at high frequencies where the modal density is increased might not be changed very much by actuating the embedded SMA fibers, but the magnitude of the transmitted power can be changed significantly in such cases (Liang et al., 1990).

\section{Transmission loss}

As described above, the transmission loss for SMA hybrid composite plates is calculated for both the activated and inactivated plate. Figure 9 shows the transmission loss versus frequencies for an inactivated and activated plate with a

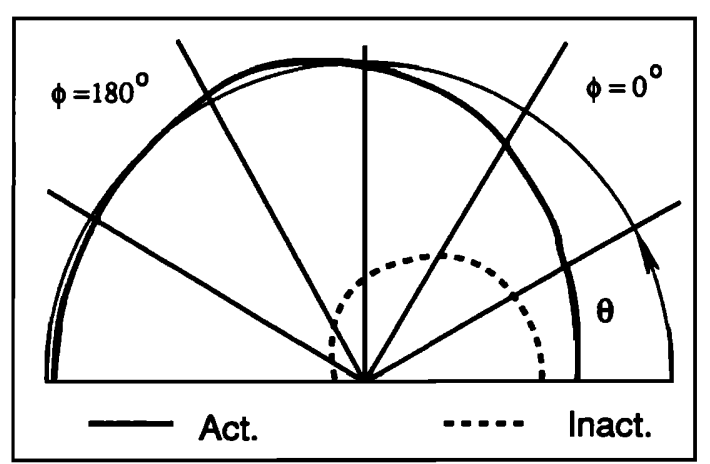

FIG. 7. Directivity pattern at $100 \mathrm{~Hz}$.

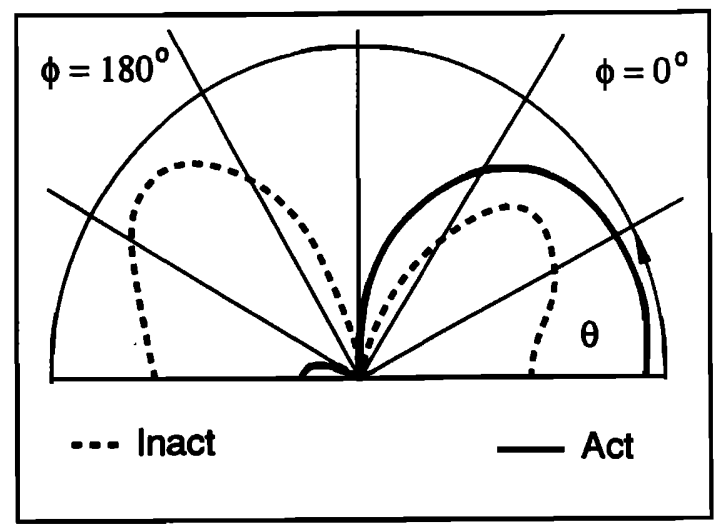

FIG. 8. Directivity pattern at $220 \mathrm{~Hz}$. 


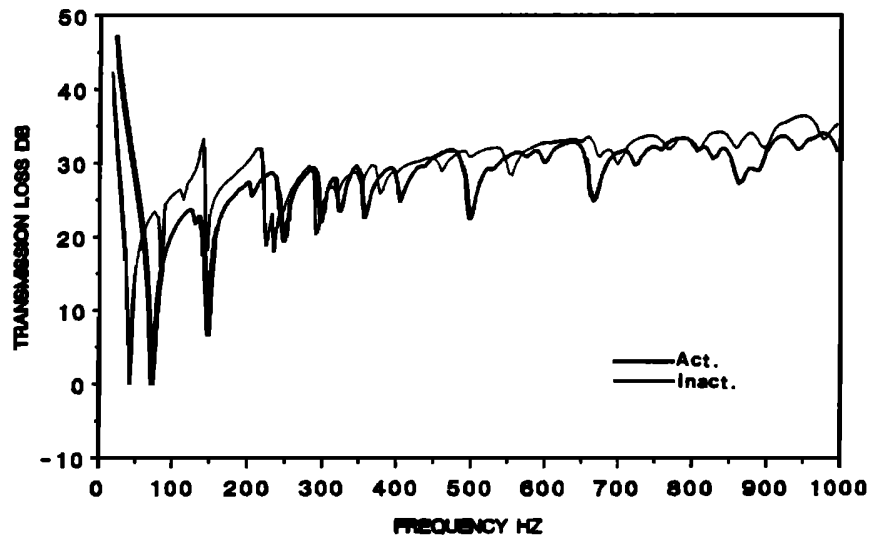

FIG. 9. Transmission loss versus frequency for low damping $(\xi=0.01)$.

damping coefficient of 0.01 . The main characteristic is a shift in the transmission loss profile to higher frequencies upon activation. However, there are two other interesting points that can be made here in reference to Fig. 9. First, for a given frequency, the transmission loss may be changed as the result of the change in the mode shape. Secondly, the overall plate stiffness can be seen to markedly influence the transmission loss characteristic. The transmission loss is reduced in an average sense over the entire frequency range being examined here and for which no explanation is apparent at this time. However, in some frequency ranges, the transmission loss may be increased by activating the SMA fibers. It is believed that as the activation degree changes (by using different activation temperatures or activating different amounts of embedded SMA fibers) the transmission loss could be controlled (either increased or reduced) in different frequency ranges. Another point is that for the same mode number, the transmission loss may also be varied greatly. For example, the fourth mode of the inactivated plate which occurs at $144.44 \mathrm{~Hz}$ has a transmission loss of 7 $\mathrm{dB}$ higher than that of the fourth mode of the activated plate. This is because the two modes have different radiation efficiencies and therefore yield different transmitted power. An examination of the results reveal that the fundamental mode shape is largely unchanged under all activation conditions considered. The main effect for activation of the SMA fibers is thus a shift in the first drop in the transmission loss curve due to an increase in the fundamental resonant frequency; there is little change in radiation due to an alteration of radiation efficiency. This suggests that activation of segments of the embedded fibers may lead to an increase in distortion of the fundamental mode and a significant increase in transmission loss due to radiation efficiency characteristics. Further work will concentrate on optimizing changes in mode shapes and stiffness in order to increase transmission loss in a controllable way.

\section{CONCLUSIONS}

This paper presented a detailed modeling and analysis scheme for shape memory alloy hybrid composites. The foundation of this analysis hierarchy is the unified constitu- tive model developed from the Helmholtz free-energy and phenomenological considerations of the martensite volume fraction. Using the constitutive model, a nonlinear dynamical model for laminated plates was developed. A refined structural acoustic analysis method was also described which solves the problem keeping the essential geometric and dynamic elements intact; namely using the "composite" modes rather than the assumed characteristic modes. This analysis scheme also showed that there is, in fact, a displacement mode coupling even though the modes are themselves orthogonal. Two new coefficients were introduced, mode transformation coefficient, and the displacement mode coefficient, which are not only useful to determine the effect of actively varying the structural dynamic characteristics of a structure but may be useful for optimization techniques. The numerical results presented show that shape memory alloy hybrid composites are effective and versatile adaptive material systems for active structural acoustic and vibration control.

\section{ACKNOWLEDGMENTS}

The authors gratefully acknowledge the support for this work by the Office of Naval Research Young Investigator Program, ONR N00014-88-0566 and ONR Grant N00014-88-0721.

Barker, D. K. (1989). "Active Dynamic Response Tuning of Adaptive Composites Utilizing Embedded Nitinol Actuators," M. S. thesis, Department of Mechanical Engineering, Virginia Polytechnic Institute and State University.

Claus, R. O., McKeeman, J. C., May, R. G., and Bennett, K. D. (1989), "Optical Fiber Sensors and Signal Processing for Smart Materials and Structures Applications," in Smart Materials, Structures and Mathematical Issues (Technomic, Lancaster, PA), pp. 29-38.

Dimitriadis, E. K., Fuller, C. R., and Rogers, C. A. (1989). "Piezoelectric Actuators for Distributed Noise and Vibration Excitation in Thin Plates," in Failure Prevention and Reliability-1989, ASME, DE-Vol. 16, pp. 223-234 (accepted for publication in J. Vibration Acoust.).

Fuller, C. R., Rogers, C. A., and Robertshaw, H. H. (1989). "Active Structural Acoustic Control with Smart Structures," in Proceedings of SPIE Conference 1170 on Fiber Optic Smart Structures and Skins II (Boston, MA).

Hagood, N. W., Crawley, E. F., de Luis, J., and Anderson, E. H. (1989). "Development of Integrated Components for Control of Intelligent Structures," in Smart Materials, Structures and Mathematical Issues (Technomic, Lancaster, PA), pp. 80-104.

Jia, J., and Rogers, C. A. (1989a). "Formulation of a Laminated Shell Theory Incorporating Embedded Distributed Actuators," Adaptive Structures, ASME, AD-Vol. 15, pp. 25-34.

Jia, J., and Rogers, C. A. (1988b). "Formulation of a Mechanical Model for Composites with Embedded SMA Actuators," Failure Prevention and Reliability-1989, ASME, DE-Vol. 16, pp. 203-210.

Jia, J., and Rogers, C. A. (1989c). "Shells with Embedded Shape Memory Alloy Actuators," in Proceedings of the 1989 International Composites Conference, Beijing, China.

Liang, C., Jia, J., and Rogers, C. A. (1989). "Behavior of Shape Memory Alloy Reinforced Plates-Part II: Results," in Proceedings of the 30th Structures, Structural Dynamics and Materials Conference, AIAA 891331, Vol. 4, pp. 1504-1513.

Liang, C., Rogers, C. A., and Fuller, C. R. (1990). "Active Transmission and Radiation Analysis of Adaptive Shape Memory Alloy Reinforced Laminated Plates," J. Sound Vib. (to be published).

Liang, C., and Rogers, C. A. (1990). "A One-Dimensional Thermomechanical Constitutive Relationship of Shape Memory Materials," in Proceedings of the 31st Structures, Structural Dynamics and Materials Conference, AIAA 90-1027.

Meirovitch, L., and Norris, M. A. (1984). "Vibration Control," in Proceed- 
ings of InterNoise 84, pp. 447-482.

Müller, I. (1979). “A Model for a Body with Shape Memory,” Arch. Rat. Mech. Anal. 70, 61-77.

Müller, I. (1985). "Pseudoelasticity in Shape Memory Alloys-an Extreme Case of Thermoelasticity," IMA Preprint No. 169.

Rogers, C. A., and Robertshaw, H. H. (1988a). "Development of a Novel Smart Material," ASME Paper 88-WA/DE-9.

Rogers, C. A., and Robertshaw, H. H. (1988b). "Shape Memory Alloy Reinforced Composites," Engineering Science Preprints 25, ESP25.88027, Society of Engineering Sciences.

Rogers, C. A., Liang, C., and Barker, D. K. (1989). "Dynamic Control Concepts Using Shape Memory Alloy Reinforced Plates," in Smart Materials, Structures and Mathematical Issues (Technomic, Lancaster, PA), pp. 39-62.

Rogers, C. A., Liang, C., and Jia, J. (1989). "Behavior of SMA Reinforced Composite Plates, Part 1: Model Formulations and Control Concepts," in Proceedings of the 30th Structures, Structural Dynamics and Materials Conference, AIAA Paper 89-1389; (American Institute of Aeronautic Astronautics, Mobile, Alabama).

Rogers, C. A. (1990). "Active Vibration and Structural Acoustic Control of Shape Memory Alloy Hybrid Composites: Experimental Results," in Proceedings of the International Congress on Recent Developments in Air and Structural Borne Sound and Vibration (to be published).

Rogers, C. A., Fuller, C. R., and Liang, C. (1990). "Active Control of
Sound Radiation from Panels Using Embedded Shape Memory Alloy Fibers," J. Sound Vib. 136, 164-170 (1990).

Roussos, L. A. (1985). "Noise Transmission Loss of a Rectangular Plate in an Infinite Baffle," NASA Technical Paper 2398.

Sato, Y., and Tanaka, K. (1988). "Estimation of Energy Dissipation in Alloys due to Stress-induced Martensitic Transformation," Res. Mech. 23, 381-393.

Sato, Y., Tanaka, K., and Kobayshi, S. (1986). "Pseudoelasticity and Shape Memory Effect Associated with Stress-induced Martensitic Transformation: A Thermomechanical Approach," Trans. Jpn. Soc. Aero. \& Space Sci. 28.

Saunders, W. R., Robertshaw, H. H., and Rogers, C. A. (1990). "Experimental Studies of Structural Acoustic Control for a Shape Memory Alloy Composite Beam," in Proceedings of the 31st Structures, Structural Dynamics and Materials Conference, AIAA 90-1090.

Tanaka, K., and Nagaki, S. (1982). "A Thermomechanical Description of Materials with Internal Variables in the Process of Phase Transformation," Ingenieur-Arch. 51, 287-299.

Tanaka, K., and Iwasaki, R. (1985). "A Phenomenological Theory of Transformation Superplasticity," Eng. Fracture Mech. 21 (4), 709-720.

Tanaka, K. (1986). "A Thermomechanical Sketch of Shape Memory Effect: One-Dimensional Tensile Behavior," Res. Mech. 18, 251-263.

Whitney, J. M. (1987). Structural Analysis of Laminated Anisotropic Plates (Technomic, Lancaster, PA). 